\title{
Comparison of Analgesic Effect of Piroxicam and Transcutaneous Electrical Nerve Stimulation Therapy on Pain associated with Orthodontic Separator Placement: A Single Blind Randomized Controlled Trial
}

\author{
${ }^{1}$ Asavari L Desai, ${ }^{2}$ Nandita Shenoy, ${ }^{3}$ Srikant Natarajan, ${ }^{4}$ Supriya Nambiar
}

\begin{abstract}
Aims and objectives: To assess and compare the efficacy of transcutaneous electrical nerve stimulation (TENS) therapy and preemptive piroxicam administration in reducing the severity of pain after orthodontic separator placement.
\end{abstract}

Materials and methods: It was a randomized, single-blind, parallel arm trial. Subjects of either gender between 18 and 30 years of age, scheduled to undergo regular orthodontic therapy with placement of two separators in each arch, were selected for this trial. The sample size consisted of 60 patients from whom prior informed consent was obtained. They were assigned randomly into two categories of 30 subjects each.

- Group I: Subjects were given piroxicam (20 mg) 1 hour before separator placement.

- Group II: Subjects were given TENS therapy immediately after separator placement.

The treatment was administered by the coinvestigator, so as to blind the principal investigator to the treatment group. Perceived pain was evaluated on chewing and fitting back teeth together at 2-, 6-, 24-, and 48-hour intervals, using the visual analog scale (VAS).

Results: Descriptive statistical analysis revealed that the VAS scores in the piroxicam group were marginally higher than the TENS group at all intervals, except at 2 hours. The difference was, however, statistically insignificant.

Conclusion: The findings of this single blind randomized, controlled trial (RCT) suggest that TENS therapy is as effective as $20 \mathrm{mg}$ piroxicam administered preemptively, in controlling pain felt due to separator placement. The TENS offers several advantages over piroxicam, as it is devoid of the inherent side effects of nonsteroidal antiinflammatory drugs (NSAIDs) like an inhibitory effect on orthodontic tooth movement and also gastric irritation.

Therefore, the use of TENS is recommended over NSAIDs for the successful management of orthodontic pain, which

\footnotetext{
${ }^{1,4}$ Department of Orthodontics, Manipal College of Dental Sciences, Mangaluru, Karnataka, India

${ }^{2}$ Department of Oral Medicine and Radiology, Manipal College of Dental Sciences, Mangaluru, Karnataka, India

${ }^{3}$ Department of Oral Pathology, Manipal College of Dental Sciences, Mangaluru, Karnataka, India

Corresponding Author: Asavari L Desai, Department of Orthodontics, Manipal College of Dental Sciences, Mangaluru Karnataka, India, Phone: +919483363045, e-mail: desaiasavari@ gmail.com/asavari.laxman@manipal.edu
}

is so critical both from the patient's and from the clinician's perspective.

Keywords: Pain, Piroxicam, Transcutaneous electrical nerve stimulation.

How to cite this article: Desai AL, Shenoy N, Natarajan S, Nambiar S. Comparison of Analgesic Effect of Piroxicam and Transcutaneous Electrical Nerve Stimulation Therapy on Pain associated with Orthodontic Separator Placement: A Single Blind Randomized Controlled Trial. World J Dent 2018; 9(4):284-290.

\section{Source of support: Nil}

\section{Conflict of interest: None}

Key message: Transcutaneous Electrical Nerve Stimulation Therapy is more effective than Piroxicam in controlling pain due to orthodontic separator placement.

\section{INTRODUCTION}

Pain is a reaction to noxious stimuli and is a highly subjective response with wide individual variations. It is an unpleasant yet inseparable part of orthodontic therapy and is associated with numerous procedures like separator placement and subsequent archwire insertion and activation. The fear of pain and discomfort is a major deterrent for many prospective orthodontic patients and is one of the most common reasons for discontinuing orthodontic treatment, making pain management a critical factor for successful treatment.

Discomfort, which can sometimes be unbearable, is often experienced by patients after even a seemingly simple procedure, such as placement of separators, which is almost always the first step of fixed orthodontic therapy. ${ }^{1-3}$ Pain increases to a peak level at approximately 24 hours following separator placement. ${ }^{4}$ Majority of patients complain of moderate-to-severe pain on biting and chewing firm or hard food. Previous studies have indicated that patients rank pain as the worst aspect of orthodontic treatment and the foremost reason for desiring to discontinue care. ${ }^{5}$ The severity and incidence of orthodontic pain is perceived to be greater than that associated with tooth extractions. ${ }^{6}$

Orthodontic forces cause disruption of blood supply to fibers attaching the tooth to the alveolar bone, which 
causes the release of prostaglandins and other inflammatory mediators into the local environment. ${ }^{7}$

Several efforts have been made in the past to mitigate orthodontic pain with NSAIDS, bite wafers, anesthetic gels, low-level laser therapy, vibratory stimulation of periodontal ligament, chewing gums, and TENS. ${ }^{8}$

As clinicians, patients often enquire if it will be necessary to take analgesics during the course of treatment and if so, which is the most effective. Previous studies have shown that preemptive doses of NSAIDs, which act by inhibiting cyclooxygenase enzymes, help to reduce the amount of pain experienced immediately posttreatment. 3,9 The NSAIDs, when given before a procedure, are absorbed and distributed by the body before tissue injury occurs. This reduces the sensitivity of nerves to certain inflammatory mediators which allows for a lower production of prostaglandins, thereby decreasing the inflammatory response. $^{10}$

Till date, the efficacy of preemptive naproxen sodium, aspirin, and ibuprofen in controlling pain has been evaluated. ${ }^{11}$

In the present study, we decided to administer piroxicam, as it offers several advantages over other regularly used NSAIDs.

Since it has a long mean half-life of 50 to 60 hours, it allows for only once-daily dosing. This may be the reason it causes significantly lower gastrointestinal irritation when compared with aspirin, ibuprofen, or naproxen sodium. It is more effective at controlling pain as compared with ibuprofen according to a previous study. ${ }^{12}$

The NSAIDs, though widely used to alleviate pain, come with their inherent side effects like gastrointestinal irritation and inhibitory effect on orthodontic tooth movement. ${ }^{13,14}$

Till date, no noninvasive, nonpharmacologic method has been clinically proven to alleviate pain experienced by orthodontic patients.

The TENS is an inexpensive, noninvasive method in which controlled, electrical pulses are applied to the site of injury. It reduces pain through both central and peripheral mechanisms with no adverse effect on tooth movement and without the risk of allergies and gastrointestinal irritation, which can be very beneficial for patients and clinicians alike.

Despite extensive research being done on the various methods available at the orthodontist's disposal to effectively manage pain, there is insufficient literature on the use of TENS therapy for controlling orthodontic pain.

This study, the first of its kind, was done to assess and compare the analgesic effect of piroxicam and TENS therapy on orthodontic pain.

\section{AIMS AND OBJECTIVES}

The aim of this article is to assess and compare the efficacy of TENS therapy and preemptive piroxicam administration in reducing the severity of pain after orthodontic separator placement.

\section{MATERIALS AND METHODS}

It was a single blind, randomized parallel arm trial for which ethical clearance from the Institutional Ethics Committee of Manipal College of Dental Sciences, Manipal University, Mangaluru, Karnataka, India was obtained (Clinical Trials of India registration number: CTRI/2017/12/010870).

Sixty-seven subjects who were scheduled to receive fixed orthodontic treatment at the Department of Orthodontics were screened for the study. Seven subjects did not fulfill the below-mentioned criteria and were hence excluded. Finally, a total of 60 subjects were enrolled in the trial, with prior informed consent (Flow Chart 1). The trial was conducted at the Department of Orthodontics, Manipal College of Dental Sciences, Mangaluru, Karnataka, India in 2017.

\section{Inclusion Criteria}

- Subject of either gender with age of at least 18 years and not older than 30 years.

- Previously untreated patients.

- Orthodontic treatment requiring the insertion of two separators in each of the four quadrants.

Flow Chart 1: CONSORT flowchart
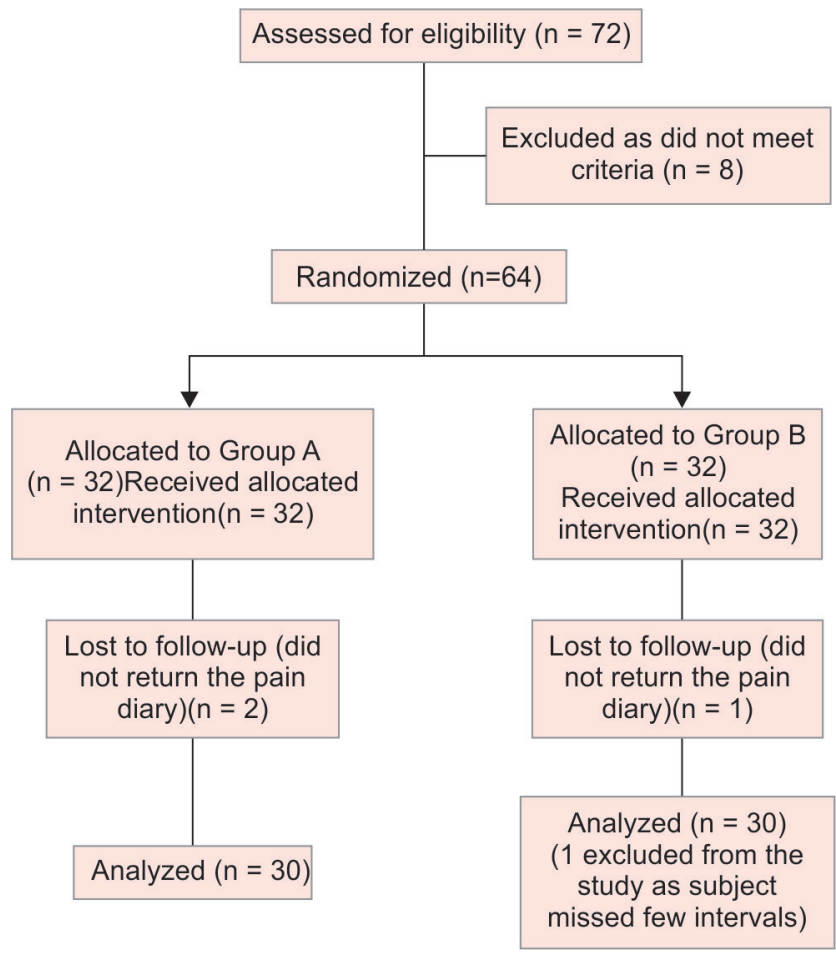


\section{Exclusion Criteria}

- Contraindications to the use of piroxicam or NSAIDS in general.

- Pregnant subjects.

- Significant medical illnesses that prevent the subject from participating in the study.

- Tooth extraction or surgical procedure before separator placement.

\section{Treatment Allocations}

The study subjects were randomly assigned to two groups of 30 subjects each.

- Group I received piroxicam (20 mg) 1 hour before separator placement.

- Group II received TENS therapy immediately after separator placement.

The above-mentioned treatment was administered by the coinvestigator so as to blind the principal investigator to the treatment group.

\section{Methods}

Subject demographics were obtained along with a thorough medical history including hypersensitivity to NSAIDs. The vitals were recorded.

Prior to separator placement, subjects were explained in detail about the VAS scale and how to rate their perceived pain consequent to separator insertion. A $10-\mathrm{cm}$ VAS was used wherein a mark at $0 \mathrm{~cm}$ was considered as "no pain at all" and $10 \mathrm{~cm}$ as "worst pain imaginable."

For subjects allocated to group I, separators were inserted 1 hour after the piroxicam administration. Two separators (Ormco, Glendora, CA) were inserted in each quadrant.

The patients allotted to group II were given TENS therapy.

The TENS unit comprised of amplitude knobs, pulse, an on/off switch, a 3-volt battery, and a pair of electrodes. A conductive gel was applied at the site of placement after which the electrodes were placed over the painful region on the cheek corresponding to the molar region on the left and right sides, in the upper and lower arches (Fig. 1).

A frequency of $50 \mathrm{~Hz}$ with a pulse width of $0.5 \mathrm{msec}$ at 0 to $60 \mathrm{~mA}$ was used. The intensity was gradually increased by turning the corresponding knob, till the patient felt a tingling sensation due to the pulse. It was further increased slowly to the point where the patient experienced maximum comfort. The intensity was maintained constant at this point and the pulse rate was increased to 2 and then to 5 .

Each subject underwent this treatment for 20 minutes after which the intensity was decreased to a minimum and then the unit was switched off.

Following this, subjects were to record the discomfort they perceived when clenching/fitting back teeth together

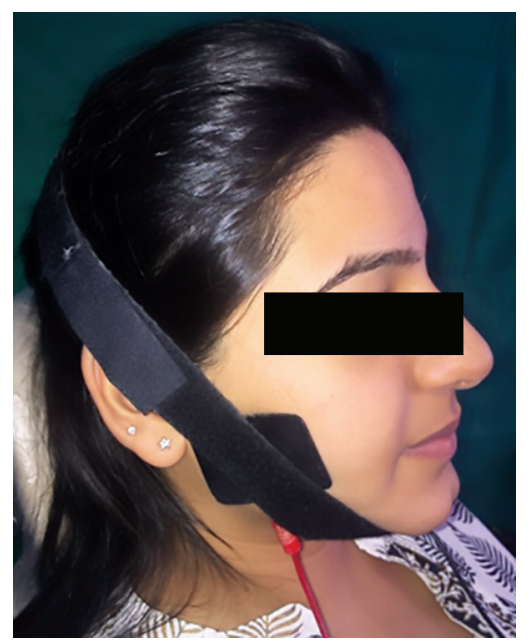

Fig. 1: TENS therapy being administered to a patient

and on chewing, at intervals of 2, 6, 24 and 48 hours after separator placement in the VAS pain diary. All the patients were instructed to return their pain diaries at the next appointment which was scheduled after 2 days, for banding of the molars. Patients who missed recording perceived pain for a few intervals were excluded from the study.

\section{Statistical Analysis}

Statistical Package for the Social Sciences (version 20.0, SPSS Inc., Chicago, Illinois, USA) was used to perform the statistical analyses. Descriptive analyses were done for assessing the pain scores at different time intervals in both the experimental groups. A repeated-measures analysis of variance (ANOVA) test with post hoc Bonferroni test was done to assess the difference in VAS scores between both the groups, across each time period; p-values less than 0.001 were regarded as statistically significant.

\section{RESULTS}

There was no statistically significant difference between the mean ages between both the groups (Graph 1). The

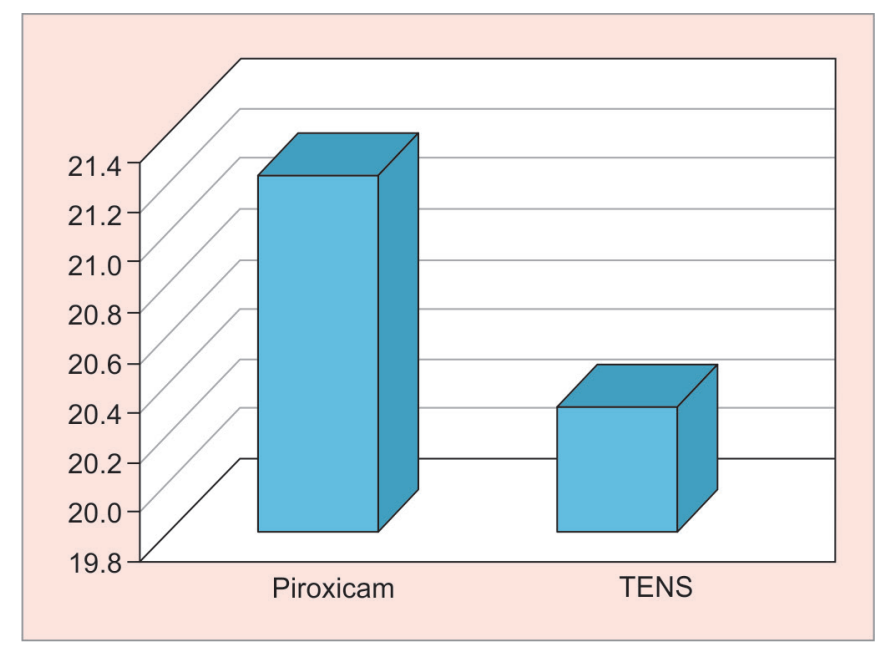

Graph 1: Age distribution among both groups 
Comparison of Analgesic Effect of Piroxicam and TENS Therapy on Pain

\begin{tabular}{|c|c|c|c|c|}
\hline & & Chewing & Fitting back teeth & Fitting front teeth \\
\hline & Group & Mean \pm Std. deviation & Mean \pm Std. deviation & Mean \pm Std. deviation \\
\hline \multirow[t]{3}{*}{2 hours } & Piroxicam & $2.3 \pm 1.86$ & $1.77 \pm 1.942$ & $1.2 \pm 1.789$ \\
\hline & Tens & $2.8 \pm 2.355$ & $2.4 \pm 2.061$ & $1.47 \pm 1.795$ \\
\hline & Total & $2.55 \pm 2.119$ & $2.08 \pm 2.011$ & $1.33 \pm 1.782$ \\
\hline \multirow[t]{3}{*}{6 hours } & Piroxicam & $3.4 \pm 2.581$ & $3.2 \pm 2.976$ & $1.93 \pm 2.612$ \\
\hline & Tens & $3.37 \pm 2.356$ & $3.83 \pm 2.601$ & $2.47 \pm 2.33$ \\
\hline & Total & $3.38 \pm 2.45$ & $3.52 \pm 2.789$ & $2.2 \pm 2.469$ \\
\hline \multirow[t]{3}{*}{24 hours } & Piroxicam & $4.43 \pm 2.944$ & $3.87 \pm 2.849$ & $2.73 \pm 3.028$ \\
\hline & Tens & $4.23 \pm 2.738$ & $3.53 \pm 2.569$ & $3.13 \pm 2.813$ \\
\hline & Total & $4.33 \pm 2.82$ & $3.7 \pm 2.695$ & $2.93 \pm 2.905$ \\
\hline \multirow[t]{3}{*}{48 hours } & Piroxicam & $4.37 \pm 2.93$ & $4.4 \pm 3.169$ & $2.87 \pm 2.837$ \\
\hline & Tens & $4.07 \pm 2.97$ & $3.5 \pm 2.515$ & $2.43 \pm 2.501$ \\
\hline & Total & $4.22 \pm 2.929$ & $3.95 \pm 2.873$ & $2.65 \pm 2.661$ \\
\hline \multirow[t]{2}{*}{ Time } & $\mathrm{F}$ & 14.653 & 17.378 & 13.592 \\
\hline & $p$-value & $<0.001$ & $<0.001$ & $<0.001$ \\
\hline \multirow[t]{2}{*}{ Time with group } & $\mathrm{F}$ & 0.675 & 3.549 & 1.289 \\
\hline & $p$-value & 0.512 & 0.028 & 0.28 \\
\hline
\end{tabular}

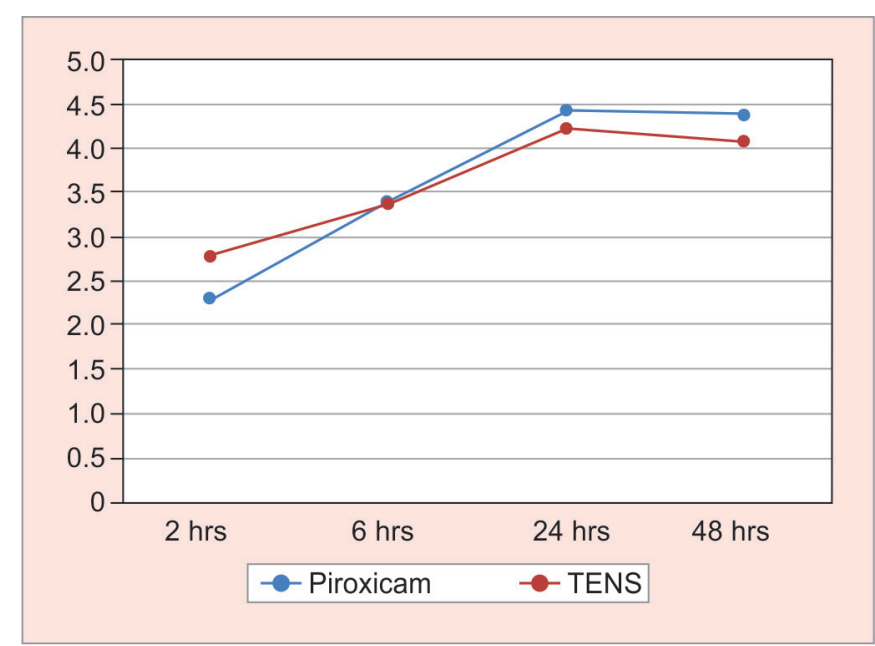

Graph 2: VAS on chewing

tests show that there was increase in VAS score from 2 to 24 hours and tapered at 48 hours in all the scenarios. This change over time was statistically significant in all the cases with the $p$-value of $<0.001$. The difference was primarily between the 24 and 48 hours in all the cases as assessed by Bonferroni's test.

\section{Differences in Pain Experienced between the Experimental Groups on "Pain on Chewing"}

The statistical analysis showed that there was an increase in VAS scores from 2 to 24 hours after which it decreased in both the groups. An interesting observation was that the VAS scores in the TENS group were lesser compared with the piroxicam group at all intervals, except at 2 hours. The difference, however, was not statistically significant, as the $\mathrm{p}$-value was $>0.001$ (Table 1 and Graph 2).

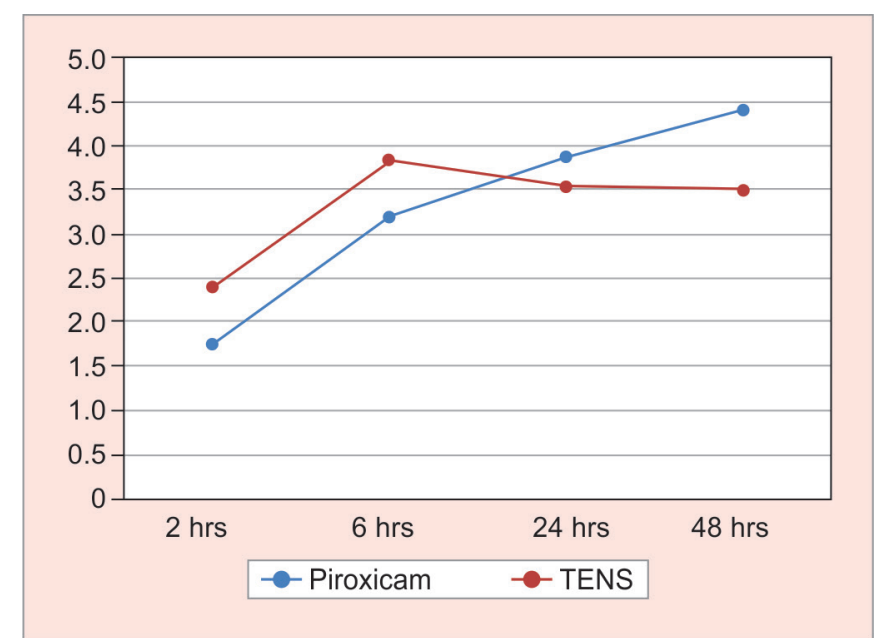

Graph 3: VAS on fitting back teeth together

\section{Differences in Pain Experienced between the Experimental Groups on "Pain on Fitting the Back Teeth"}

The results of the statistical analysis revealed that the pain experienced by subjects in the piroxicam group increased gradually from 2 to 48 hours. In the TENS group, however, the VAS scores decreased significantly after 6 hours itself and the difference was statistically significant. A comparison of the scores between the two groups showed that the scores in the TENS group were less than those in the piroxicam group after 6 hours, though the difference was statistically insignificant (Table 1 and Graph 3).

\section{DISCUSSION}

This trial was designed to evaluate and compare the analgesic efficacy of TENS therapy and piroxicam on orthodontic pain, using a sample size of 60 individuals 
who were scheduled to undergo fixed appliance therapy. As there is no objective method available to assess the severity of perceived pain, the VAS was selected, as it has been proven to be the most accurate and reliable tool to measure subjective experiences like pain. ${ }^{15}$

Piroxicam was chosen over ibuprofen, which is the most commonly prescribed analgesic, as it offers several significant advantages. It has a longer mean half life which therefore prolongs its duration of action and permits once-daily dosing. Also, a previous trial conducted by Kohli and Kohli ${ }^{16}$ which compared the analgesic efficacy of piroxicam, ibuprofen, and a placebo showed that piroxicam controlled pain more effectively compared with ibuprofen and the placebo.

However, a major concern with the use of NSAIDs to manage orthodontic pain is the likelihood of interference with tooth movement due to the inhibition of cyclooxygenase activity and thus prostaglandin production.

The TENS therapy is an inexpensive, noninvasive method of alleviating both acute and chronic pain. Since it is nonpharmacologic, there is no risk of side effects like allergic reaction to medication or inhibiting orthodontic tooth movement. Pain reduction with TENS is attributed to the electrical stimuli, pressure, and touch impulses which arrive faster at the levels of the spinal cord in the substantia gelatinosa of the dorsal horn and in the higher levels of the central nervous system than the pain impulses and "close the gate" for pain impulses, resulting in a suppression of pain signals. The TENS also causes activation of endogenous analgesic systems involving opiate-like peptides, such as endorphins, thereby increasing their plasma levels. ${ }^{17}$

In dentistry, TENS was first used for the treatment of myofascial pain dysfunction. The TENS was used by Roth and Thrash ${ }^{18}$ to assess its effect on periodontal pain associated with the placement of orthodontic separators inserted on the mesial and distal of upper first molars, in 45 adult patients. Patients who were given TENS reported significant decrease in pain on VAS at the 24-, 36-, and 48-hour assessment periods, whereas the control group experienced postadjustment pain even after a period of 60 hours. A literature search revealed that this was the only study which assessed the effect of TENS on orthodontic pain. More recently, TENS has been used during pulpotomy, cavity preparation, and tooth extraction. ${ }^{19}$

In spite of randomization, the gender distribution was unequal, with more males than females in both the groups (Graph 4). Previous studies have shown that there is no difference in perceived pain during orthodontic treatment between the sexes..$^{20}$ Gender discrimination was therefore not included, and males and females were evaluated together with the rest of the data.

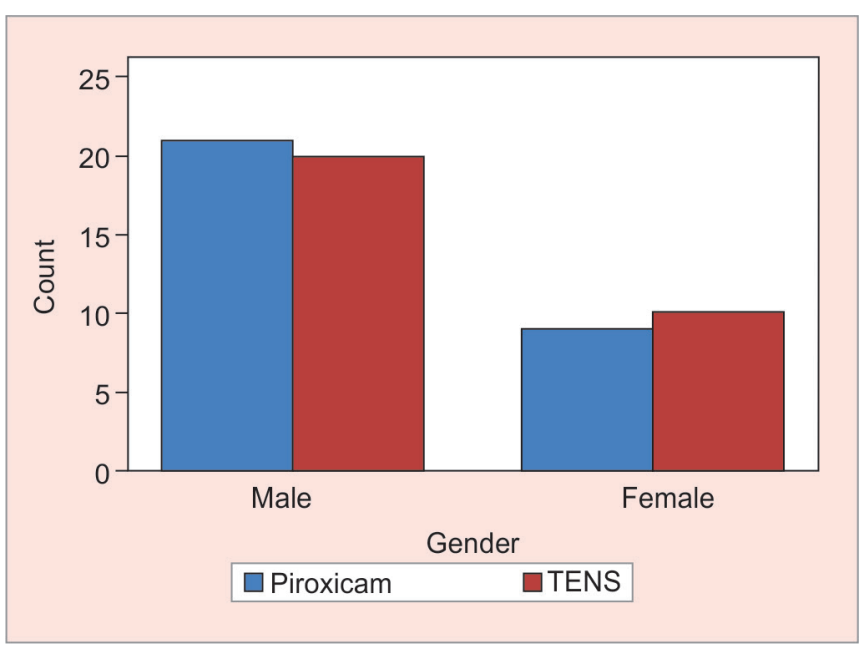

Graph 4: Gender distribution in both groups

The findings of this trial indicated that the pain experienced by the patients in both the treatment groups gradually increased 2 hours after separator placement and peaked at the 24-hour interval, after which it declined. This corroborates the findings of previous studies by Law et al, ${ }^{3}$ and Kohli and Kohli. ${ }^{16}$

The greatest reported pain was observed on chewing rather than on fitting back teeth together or fitting front teeth together, which corroborates the findings of previous studies. This could be due to the fact that orthodontic pain is caused by the compression and inflammation of the periodontal ligament, and maximum compression occurs during chewing. ${ }^{21}$

The patients who were administered $20 \mathrm{mg}$ of piroxicam 1 hour before separator placement exhibited slightly lower levels of pain than those that were given TENS therapy up to the 24-hour interval. Interestingly, after the 24-hour interval, subjects who had been given TENS showed mildly lower levels of pain across all the three categories, i.e., pain on chewing, pain on fitting back teeth together, and pain on fitting front teeth together. This may be attributed to the fact that the therapeutic plasma levels of piroxicam gradually begin to decline after 24 hours. ${ }^{22}$ With respect to TENS-induced analgesia, different studies have reported varied durations of action, lasting anywhere between $2^{23}$ and 18 hours. ${ }^{24}$ This variation reflects a natural fluctuation in symptoms and the patient's expectation of treatment duration rather than specific TENS-induced effects. It is believed that postTENS analgesia is longer for acupuncture-like TENS than for conventional TENS and this is supported by initial findings in experimental studies. ${ }^{25}$ However, more work is needed to establish the time course of analgesic effects of different types of TENS.

The differences in pain levels experienced by the patients in both the groups were not statistically significant 
and are hence likely to be clinically insignificant as well. This finding is of a great significance, as it lends support to the idea that TENS can replace the traditionally used NSAIDs as a method of controlling pain associated with orthodontic therapy. It will be highly beneficial for both patients and clinicians, as it is devoid of any of the several side effects that are inherent to the use of NSAIDs. There are certain contraindications for TENS therapy and should be avoided in patients with cardiac pacemakers, cerebrovascular problems, epilepsy, and pregnancy. $^{26}$

However, the advantages of TENS therapy far outweigh the disadvantages associated as it is noninvasive, safe, and effective, which lead to positive acceptance by the patients.

\section{CONCLUSION}

The findings of this single blind RCT suggest that TENS therapy is as effective as $20 \mathrm{mg}$ piroxicam administered preemptively, in controlling pain experienced due to orthodontic separator placement.

The use of TENS is recommended over NSAIDs due to its several advantages, both from the patient's and from the clinician's perspective.

\section{CLINICAL SIGNIFICANCE}

Patients complain of significant pain postorthodontic separator placement which prompts the injudicious use of analgesics by both the doctor and patients. The use of pharmacological agents for alleviating pain comes with its inherent drawbacks of gastric irritation and potential for inhibiting orthodontic tooth movement. The TENS, on the contrary, is a nonpharmacological and safe method to control pain, bereft of the above-mentioned side effects. The results of this trial suggest that TENS can be effectively used clinically instead of analgesics, to reduce the patient's discomfort.

\section{REFERENCES}

1. Bergius M, Berggren U, Kiliaridis S. Experience of pain during an orthodontic procedure. Eur J Oral Sci 2002 Apr;110(2): 92-98.

2. Ngan P, Wilson S, Shanfeld J, Amini H. The effect of ibuprofen on the level of discomfort in patients undergoing orthodontic treatment. Am J Orthod Dentofacial Orthop 1994 Jul;106(1): 88-95.

3. Law SL, Southard KA, Law AS, Logan HL, Jakobsen JR. An evaluation of preoperative ibuprofen for treatment of pain associated with orthodontic separator placement. Am J Orthod Dentofacial Orthop 2000 Dec;118(6):629-635.

4. Bernhardt MK, Southard KA, Batterson KD, Logan HL, Baker KA, Jakobsen JR. The effect of pre-emptive and/or postoperative ibuprofen therapy for orthodontic pain. Am J Orthod Dentofacial Orthop 2001 Jul;120(1):20-27.
5. Oliver RG, Knapman YM. Attitude to orthodontic treatment. Br J Orthod 1985 Oct;12(4):179-188.

6. Jones ML, Chan C. Pain in the early stages of orthodontic treatment. J Clin Orthod 1992 May;26(5):311-313.

7. Urquhart E. Analgesic agents and strategies in the dental pain model. J Dent 1994 Dec;22(6):336-341.

8. Hussain AS, Toubity MJ, Elias WY. Methodologies in orthodontic pain management: a review. Open Dent J 2017 Aug;11:492-497.

9. Mehlisch DR. The efficacy of combination analgesic therapy in relieving dental pain. J Am Dent Assoc 2002 Jul;133(7): 861-871.

10. Jackson DL, Moore PA, Hargreaves KM. Preoperative nonsteroidal anti-inflammatory medication for the prevention of postoperative dental pain. J Am Dent Assoc 1989 Nov;119(5):641-647.

11. Polat O, Karaman AI, Durmus E. Effects of preoperative ibuprofen and naproxen sodium on orthodontic pain. Angle Orthod 2005 Sep;75(5):791-796.

12. Furst, DE.; Munster, T. Nonsteroidal anti-inflammatory drugs, disease modifying anti rheumatic drugs, non-opiod analgesics and drugs used in gout. In: Katzung BG, editor. Basic and clinical pharmacology. 8th ed. New York: Lange-McGraw Hill; 2001. p. 596-624.

13. Ashkenazi M, Berlin-Broner Y, Levin L. Pain prevention and management during orthodontic treatment as perceived by patients. Orthodontics(Chic) 2012 Mar;13(1):e76-e81.

14. Krasny M, Zadurska M, Cessak G, Fiedor P. Analysis of effect of non-steroidal anti-inflammatory drugs on teeth and oral tissues during orthodontic treatment. Report based on literature review. Acta Pol Pharm 2013 May-Jun;70(3): 573-577.

15. Sriwatanakul K, Kelvie W, Lasagna L, Calimlim JF, Weis OF, Mehta G. Studies with different types of visual analog scales for measurement of pain. Clin Pharmacol Ther 1983 Aug;34(2):234-239.

16. Kohli SS, Kohli VS. Effectiveness of Piroxicam and Ibuprofen premedication on orthodontic patients' pain experiencesa randomized, controlled trial. Angle Orthod 2011 Nov;81(6): 1097-1102.

17. Wessberg GA, Carroll WL, Dinham R, Wolford LM. Transcutaneous electrical stimulation as an adjunct in the management of myofascial pain-dysfunction syndrome. J Prosthet Dent 1981 Mar;45(3):307-314.

18. Roth PM, Thrash WJ. Effect of transcutaneous electrical nerve stimulation for controlling pain associated with orthodontic tooth movement. Am J Orthod 1986 Aug;90(2):132-138.

19. Kasat V, Gupta A, Ladda R, Kathariya M, Saluja H, Farooqui AA. Transcutaneous electric nerve stimulation (TENS) in dentistry—a review. J Clin Exp Dent 2014 Dec;6(5):e562-e568.

20. Ngan P, Kess B, Wilson S. Perception of discomfort by patients undergoing orthodontic treatment. Am J Orthod Dentofacial Orthop 1989 Jul;96(1):47-53.

21. Najafi HZ, Oshagh M, Salehi P, Babanouri M, Torkan S. Comparison of the effects of preemptive acetaminophen, ibuprofen and meloxicam on pain after separator placement: a randomized clinical trial. Prog Orthod 2015 Oct;16:34.

22. Eandi M, della Pepa C, Rubinetto MP. Piroxicam in analgesia. Clin Ter 1991 Jan;136(2):107-135.

23. Johnson MI, Ashton CH, Bousfield DR, Thompson JW. Analgesic effects of different pulse patterns of transcutaneous 
electrical nerve stimulation on cold-induced pain in normal subjects. J Psychosom Res 1991;35(2-3):313-321.

24. Augustinsson L, Carlsson C, Pellettieri L Transcutaneous electrical stimulation for pain and itch control. Acta Neurochirurgica 1976;33:342.
25. Johnson MI, Ashton CH, Thompson JW. Analgesic effects of acupuncture like TENS on cold pressor pain in normal subjects. Eur J Pain 1992;13:101-108.

26. Quarnstrom F. Electronic dental anesthesia. Anesth Prog 1992 Feb;39(4-5):162-177. 